stiffness, reduced speech output and confusion. Only Patient 3 gave a history of genital ulceration which had occurred during her initial clinical presentation, with no subsequent genital recurrences. While the role of long-term antiviral therapy is well-established in the prevention of genital recurrences, evidence for its efficacy in recurrent HSV2 meningitis is equivocal. Challenges encountered in managing these patients illustrate: (i) the protean neurological manifestations of HSV2 infection; (ii) the need for better evidence supporting acute and prophylactic antiviral treatment to prevent neurological recurrence; and (iii) the need to develop novel therapeutic approaches to prevent long term morbidity.

\title{
HERPES SIMPLEX TYPE 2 ASSOCIATED NEUROLOGICAL SYNDROMES
}

Ashvini Keshavan, ${ }^{1}$ David Breen, ${ }^{1}$ Cecilia Theodore, ${ }^{2}$ Anjum Misbahuddin, ${ }^{1}$ Rajith De Silva'. 'Essex Centre for Neurological Sciences; ${ }^{1}$ Department of Genitourinary Medicine; Barking, Havering and Redbridge University Hospitals NHS Trust

\subsection{6/jnnp-2014-309236.118}

Three women with neurological syndromes associated with Herpes Simplex type 2 (HSV2), diagnosed by PCR, are presented. Patient 1 had lymphocytic meningitis with no antecedent or concurrent genital symptoms. Patient 2 had chronic lymphocytic meningitis in the context of immunosuppressive medication and Mollaret cells in the cerebrospinal fluid. She has persistent neurological symptoms despite prolonged treatment with intravenous acyclovir, cessation of immunosuppression and clearance of viral nucleic acid from the cerebrospinal fluid. Patient 3 had a 6 year history of a recurrent vesicular eruption in a dermatomal distribution, accompanied by headache, neck 